International Journal of Biomedicine | June 2021 - Volume 11, Issue Suppl_1: Abstracts from the Third Russian International Conference "Cryo-electron microscopy 2021: achievements and prospects"

POSTER ABSTRACT PRESENTATIONS

SESSION TITLE: STRUCTURE AND FUNCTIONS OF THE TRANSCRIPTION AND TRANSLATION APPARATUS OF THE CELL

DOI: 10.21103/IJBM.11.Suppl_1.P19

\title{
Abstract P-19: 15-Microsecond Molecular Dynamics Simulations of Nucleosome Show Structural Heterogeneity and Plasticity
}

$\underline{\text { Grigoriy A. Armeev }}{ }^{1}$, Anastasiia S. Kniazeva ${ }^{1}$, Galina A. Komarova ${ }^{3}$, Mikhail P. Kirpichnikov ${ }^{1,4}$, Alexey K. Shaytan ${ }^{1,2,5}$

${ }^{1}$ Department of Biology, Lomonosov Moscow State University, Moscow, Russia

${ }^{2}$ Sirius University of Science and Technology, Sochi, Russia

${ }^{3}$ Department of Physics, Lomonosov Moscow State University, Moscow, Russia

${ }^{4}$ Shemyakin-Ovchinnikov Institute of Bioorganic Chemistry, Russian Academy of Sciences, Moscow, Russia

${ }^{5}$ Bioinformatics Lab, Faculty of Computer Science, HSE University, Moscow, Russia

Background: Nucleosomes are basic units of chromatin organization, resembling spools with $\sim 150$ base pairs of DNA wrapped around the octamer of histone proteins. They play a crucial role in chromatin compactization and gene expression. Currently, there are more than 340 structures of nucleosomes and their complexes with proteins in the protein data bank, 159 of them are made with cryoEM, 60 of those in 2020 and later. It is clear that cryoEM will soon yield even more structures of nucleosomes with different histone variants, mutations, DNA sequences, and interacting proteins. Despite the variety, the majority of the aforementioned structures look very similar. This is due to the fact that most of the models are built on the basis of very similar crystal structures. However, the dynamics of nucleosomes are crucial for understanding the mechanisms that govern the chromatin functions. Computational methods can supplement experimental approaches and recreate the dynamic conformational landscape of nucleosomes from initial static structures. We present an all-atom molecular dynamics simulation of nucleosome core particles at a record timescale of 15 microseconds.

Methods: All-atom MD simulations were performed using GROMACS 2018 with AMBER ff14SB force field with parmbsc1 DNA and CUFIX ion 
parameters. Crystal structures with PDB IDs 1KX5 and 3LZ0 were used. Analysis was performed with custom-developed python scripts based on MDAnalysis and 3DNA. Models of chromatin fibers were built by connecting random snapshots from MD trajectories with straight linker segments of B-DNA of different lengths.

Results: We observed the inner dynamics of histone octamer, which covers the conformational space of the most deformed structures reported by cryoEM. We showed that histone dynamics play important role in DNA mobility, allowing for twist-defects propagation.

Conclusion: We observed unprecedented unwrapping of nucleosomal DNA with truncated histone tails. Through multi-scale modeling, we showed that such unwrapping alone is crucial for nucleosomal fibers geometry and elastic properties.

Key Words: nucleosome $・$ chromatin $\bullet$ molecular dynamics

This work was supported by the Russian Science Foundation (Grant No. 1874-10006)(MD simulations and analysis), RFBR grants (Grant No. 20-3470039) (supranucleosome structure modeling), (Grant No. 19-34-51053) (development of protein-DNA analysis algorithms) and by the Interdisciplinary Scientific and Educational School of Moscow University "Molecular Technologies of the Living Systems and Synthetic Biology". A.K.S is supported by the HSE University Basic Research Program. The research was carried out using the equipment of the shared research facilities of HPC computing resources at Lomonosov Moscow State University.

*Corresponding author: Grigoriy Armeev.E-mail: armeev@intbio.org

International Journal of Biomedicine. 2021;11 Suppl 1: S19-20.

doi: 10.21103/IJBM.11.Suppl_1.P19

(C)2021 International Medical Research and Development Corporation 